\title{
Research on Applications of Core Strength Training in Physical Education in Universities
}

\author{
Yong Liü, a \\ ${ }^{1}$ Henan Quality Polytechnic, Pingdingshan, 467000, China \\ ${ }^{a}$ email
}

Keywords: Core Strength, Strength training, Physical Education

\begin{abstract}
The core strength training is a new idea of strength training which was transplanted from the rehabilitation field into the competitive sports training in1990s. At present, the core strength has been widely used in swimming training, badminton training and athletics training in colleges and universities of our country. This paper discusses the concept, application items and application methods of core strength training, which aims to improve the core strength understanding of relative personnel.
\end{abstract}

\section{Introduction}

Core strength training is the training of the strength, stability and balance of the body, trunk, core, muscles and their small groups of muscles. Core strength training usually includes the core stability and core strength training of the two parts, the two are complementary, core strength is the foundation of core stability and core stability is the theoretical source of core strength training methods and means. In practice, many athletes are only concerned with abdominal surface muscle training, such as the rectus abdominis, in order to stabilize the trunk of the body. But rectus abdominis is mainly responsible for the body trunk end to the center of the body near, and cannot support the lower limb trunk movement, the trunk of the lower limb requires the participation of the core muscles. If the core strength of the athletes is relatively weak, the automatic activation of the core muscles will be poor, it is difficult to protect the stability of the trunk of the body. Athletes in the training preparation period, need to carry out special core strength training, in order to activate the core muscle group function, protect and stabilize the back of the waist, so as to avoid sports injury. It is only through systematic training for the core muscles that the core muscles are activated in time. In order to activate the correct muscles, athletes usually need to take sitting, kneeling or lying posture approach to training, take this position to make the body in the center, the core strength training is usually fine training, need to wake up and activate specific muscle groups, which are usually with athletes in muscle the main group of strength training is different. Although the range of movement of core strength training is not large, it has significant effect on the stability of the whole body.

\section{Applications of Core Strength Training in Physical Education in Universities}

Applications of Core Strength Training in Swimming. The biggest characteristic of the sport of swimming is from out of Taiwan athletes began, the body is finished all in a state of unfixed support and instability. This makes the athletes should always change their position in the water, so they can keep balance in the water, and through the core strength training to the ability of athletes' effective formation and promotion. In the process of human motion in multiple muscle groups in the body and joints are involved at the same time, and in the kinematics study, how can the human body shrinkage of different muscles and joint kinematic chains and organic integration, well formed a composite mechanics, which can make the athletes in the water force better, it has become the most concern for sports. In the process of swimming, in the past that are more technical points such as the limbs of athletes, lower limbs kick, upper limb stroke technique and so on, but in the process of attention to some limbs were ignored in the trunk can be used in the first tour way is appropriate to increase the athletes water exercises, such as ground ball exercise mat, practice and so on. Secondly through 
training can enhance the control ability of athletes in the water body, such as rolling, appropriate in water by spinal forces to move forward and so on. Through the athlete has the good core strength, only then can make it in the movement process, the trunk obtains the more stable support, thus causes the limbs to obtain the better technical movement to display. In swimming, coordination nor emphasize athlete force, good coordination of the only athlete has to force, to ensure it can keep good technical movements in the water, and in the promotion of good technical action that itself can be in the form of high speed and low energy consumption to obtain better swimming performance.

Applications of Core Strength Training in Badminton. Badminton ball technology is divided into four parts: preparation, racket, racket, ball. Ready for action includes body stance and hand racket will be held in what position; the racket ball is ready to prepare for the swing, is also a potential energy accumulation is a process of force swing. The ball hit the ball when the final command take action, the player according to tactical needs, by controlling the speed of swing angle of the racket face, make the ball in different flight routes to other sites, after hitting the hitter, with action to recover homeopathic racket, restore to the ready state in general, hit the ball hit back than the technical requirements of the front the ball is exquisite, the technical requirements of highly skilled, hitting to steady, so the only possibility to win; and the midfield ball for the requirement of relative backcourt frontcourt hitting speed and strength will hit the ball than before. It can be concluded that the core training in the practice application of the backfield will be more, relatively low drop, so this paper mainly from the backfield technical analysis, applied research on core strength training in badminton. This action is very demanding on the waist and abdomen, requiring rapid rotation in a short period of time. When the ball in front of the shoulder, forearm pronation, wrist flexion slightly closed, flash wrist force smash twist on the waist and abdomen, flexibility and strength has certain requirements. If the waist strength is not very good, so the swivel abdomen is not in place, it is difficult to complete high quality smash. According to the relevant knowledge of the core strength and the above analysis of badminton technology, badminton in the backcourt technology, compared with the field skills, more core strength exercises. From a biomechanical point of view, in any sport, the transfer of force is transmitted from the distal to the proximal part.

Applications of Core Strength Training in Athletics. In the core strength training, we can creatively show the unstable state of track and field athletes in the movement, and let the trained athletes realize the importance of controlling their stability. When the body is in motion, to maintain the stability of the body, it is necessary to mobilize the nervous system of the trunk core muscle is adjusted, so you can also train athletes perception of one's own body. With the increase of athletes' perception ability, the coordinated control ability in sports will be improved. In theory, the regulation of the core muscle seems simple, but in practice it is very difficult to successfully mobilize the central nervous system. Then, the training difficulty should be graded so as to implement the training plan step by step, which is divided into stationary and unstable states. In the steady state core strength training for athletes, so that they can keep the body posture, do some exercise can control; in the non-steady state core strength training for athletes, so that they can keep the core static, hand to hand movement and drag movement. The core functional force is a specialized ability in physiological structure and neuromuscular adaptation, which is adapted to the special technical movement structure. It is the force produced by the coordination of nerves and muscles and the coordination among multiple muscles. It is the bridge between the core stability and the special forces. Coordination of power through the core, effectively changed to special forces, so as to realize and solve in the process of training, to complete the transition and transformation between core stability training and special strength training. The special strength refers to the completion of the special action itself play strength, integrity and coordination force is more focused on the specific technical movement, functional strength of special strength can be said to be a kind of alienated. Static actions the purpose of the training is to improve the ability of early stage in the process of motion control is usually stable center of gravity core training using this way of training athletes in the training process, can feel the core parts of the body muscles in hard state. No load training is based on the static action training on the basis of the initial training feet to support single foot support, early training arms support to support a single body at rest can do some other relaxation exercises. 


\section{Application Points of Core Strength Training in Physical Education in Universities}

Training Actions Selection. As a trainer, when formulating a training program, we should develop differentiated training programs according to different athletes' basic abilities, so as to better meet the actual needs of athletes. In addition to teaching students in accordance with their aptitude, in the process of training the core strength should also be done step by step. This. According to the core idea of strength training, we can be the mode of training is mainly divided into two categories: the first category, mainly to maintain the level of athletes in the position of the backstroke and freestyle, and in another is to keep athletes in the state of the ups and downs of butterfly and moth swimming. In these two kinds of different training methods for athletes, the core force in the development of different emphasis: in the training mode, the main requirements of core strength shall be balanced, static, and body posture is more inclined to tight, flat, straight, the whole is a "static strength training. In second kinds of training methods, the main requirements of athletes should tend to change, the core parts of power ups and downs, and athletes attitude is also more emphasis on the limbs of the same force, generally speaking is a kind of "dynamic power" to practice in the actual process of training, the best guarantee of the above two types of training with alternate use, thus the athletes can be combined in one movement, each part of the body of the alternate training hall better core strength exercises and reduce fatigue. At the same time, the process should emphasize the point that the two training methods has great differences, but also have close ties, but their focus is different, but in the actual process of training athletes, also they must be effectively combined to obtain better training effect.

Training Time Arrangement. The core strength and the traditional strength training complementary advantages, to some extent, the former is the latter part of the content and useful supplement. According to the characteristics of training objects, tasks, stages and age, the proportion and timing of the two should be scientifically and rationally arranged. The body is a complete chain of power, which cannot be separated from practice. Keep your body steady while running, and keep your movements straight. In recent years, international swimming gradually showing a high tempo, short period training, which also makes in the process of carrying out the core strength training for athletes should be more reasonable arrangement for the training time, which can make the athletes get better training effect. In this regard, we can be divided into two main forms according to the different positions of the trained muscles: whole muscle training and local muscle training. In the development of local muscle strength training, athletes can be arranged to prepare activities fully, then the stability of strength training, so in this way to enable the athletes to achieve good physical stability on the ground, then you will be able to better the stability of the transfer to the stability of training in the water, and in the whole muscle strength training, it can be properly with ground forces training together, so as to obtain the balanced development of the two forces. It is suggested that two times a week after each training session. Dedicated core strength is a kind of nerve muscle, energy metabolism, physiological structure, psychological adjustment ability and special skill to improve it in power, based on the core stability ability and special needs to adapt and docking, the basic strength and functional strength obtained by coordinating force and smoothly in the special sports express, simply dynamic stability is an outstanding and powerful waist and hip contraction force. The above training is manual training, low strength, at home or outdoor can be completed. Core strength training, as an important part of system training, can not only avoid sports injuries, but also help to run. When the muscles needed for running are exercised and your hips and torso work together, you are less injured and enjoy running better.

Training Stage Division. In the preparation stage, mainly rely on athletes by overcoming some of their body weight exercises to build the core stability foundation, grasp the correct action, should follow from the inside of the principle in the application of abdominal muscles, the main purpose for core strength training phase is added to obtain stability, coordination, stimulate the deep muscles, master the correct action, and reduce and prevent injury. Note that, in the preparation stage and cannot be the core strength training as strength training, strength training routine is still the main content, emphasizing the core strength training does not mean that the resistance against strength 
training of neglect, does not mean to abandon limb strength training. Once the stability of the core muscles to consolidate in the preparation stage, we should start with the special development related to limb muscle training, because of the special skill is the key to decide the level of special skills, in the development of special skills, need special action mode combined with resistance exercise. At this point, it is necessary to carry out multi plane training, functional training, strength, coordination, acceleration, deceleration, change direction and other exercises should not be carried out alone, but the overall coordination exercises. In the athletic preparation and competition stage, the core strength training mainly focuses on the nerve activation training, and usually takes the special similar action or the load to carry on ultra-equal length training and balanced training. The usual exercises include various functional exercises performed on unstable support surfaces, in single leg support, in body tilt, and in suspension. The common characteristics of this kind of practice is that the non-steady state, the purpose is to improve the level of muscle activation, increased muscle movement of proprioceptive stimulation, the exercise of the acquired power can be effectively converted to the movement of. Through the training of stability and coordination ability, we can promote the coordination of different muscles under the control of the nervous system.

\section{Conclusion}

With the progress of sports science research, various training theories have been put forward. It makes the core strength training become a concern. Core strength training has become an effective way of training university students. It can not only improve the training efficiency of university students, but also help the rapid rehabilitation of university students after injury.

\section{References}

[1] Wen Jianfei, Gun Junjun, Effects of Core Strength Training on Balance Ability and Back Muscle Strength of Male College Students [J]. Bulletin of Sport Science \& Technology, 2016(5): 76-78.

[2] Jiang Hongbin, Research on Human Movement Core Stability and Core Strength Training [J]. Journal of Capital University of Physical Education and Sports, 2015, 27(3): 257-263.

[3] Wei Xiaofang, Liu Xie, Jiang Hongbin, Core Strength Training Theory- A New Field of Scientific Training [J]. Journal of Chengdu Sport University, 2013, 39(8): 47-51.

[4] Han Chunyuan, Wang Weixing, Cheng Bojin, Liu Shufang, Basic Issues of the Core Strength Training: The Core Area and Core Stability [J]. Journal of Tianjin University of Sport, 2012, 27(2): 117-120. 